\title{
Simulation modeling of the curvilinear motion of an industrial tractor with a differential rotation mechanism and tracking trajectory stabilization system
}

\author{
Sergey V. Kondakov ${ }^{1}$, Aleksandr A. Dyakonov ${ }^{1, *}$, and Nikita V. Dubrovskiy ${ }^{1}$ \\ ${ }^{1}$ South Ural State University, Chelyabinsk, Russia
}

\begin{abstract}
The article is devoted to the investigation of the transient processes in the hydrodynamic transmission and hydro-hydraulic turning mechanism at the entrance to the turn and turning on the spot. Boundary ground conditions are determined for the tractor to perform stepless maneuvering while performing technological operations. The algorithm of the tracking system has been formulated, which made it possible to keep the radius of the trajectory fixed by the handwheel as the external resistance to movement changes.
\end{abstract}

\section{Introduction}

Mechanisms for the rotation of caterpillar vehicles based on hydrostatic transmissions (HST) are known $[1,2]$. Basically, they are used in high-speed cars (tanks, infantry armored cars, tractors) $[3,4]$. The experience of using similar schemes of turning mechanisms is also found in slow-moving caterpillar machines $[5,6]$, although it is not so great. This is due to a noticeable rise in the cost of construction, the need to install planetary summing gears and actually HST. Feasibility and advantages of the transition to a step-less turn of industrial tractors requires justification. In part, it is set out in this article.

Disadvantages of HST are obvious - low efficiency, about $80 \%$. The advantages are not obvious: they need to be explained. First, $80 \%$ efficiency corresponds to all the turning radii, from infinity to zero, that is, from the movement close to rectilinear ("steer" to compensate for the withdrawal) before turning around in the center of gravity. With a conventional tractor, the fixed radius is only one - around the stopped track. Less often - two, for tractors with a two-stage planetary rotation mechanism. All other radii are achieved by slipping clutch, and this loss, in many cases more than $20 \%$. We emphasize that this is the cost of turning control - a complete analog of the losses in the HST turning mechanism.

Secondly, the ease of controlling the HST with a joystick or hand wheel is not achievable in the control of frictions. The friction cannot in principle provide the desired, but still stable, slip in the constant position of the control lever.

\footnotetext{
* Corresponding author: dyakonovaa@susu.ru
} 
Thirdly, the accuracy of control: it is one thing to operate the steering wheel as a car, quite another thing by friction, essentially a side brake, and hoping to "catch" some desired turning radius, which coincides with the curvature of the route. When controlling the rotation of the clutches, the driver is forced to reduce the speed of movement, and this reduces the performance of the tractor.

\section{Mathematical model}

\subsection{Structural and calculation schemes}

The kinematic diagram of the transmission of the tracked vehicle (TV) is shown in Fig. 1. The specificity of the machine under investigation is the order of the connection of the main links of the SPS with the turbine HT (SPS carrier), the HST engine (SPS sun) and the driving wheel (SPS epicycle). In none of the similar machines mentioned above, there is no such connection. This feature was reflected in (5) and (6) equations of the mathematical model. The calculated scheme of the plane-parallel motion of the TV in immovable Cartesian coordinates is shown in Fig. 2 and 3. According to the calculation scheme, the position of the machine on the plane is given by two linear coordinates of the center of gravity $\mathrm{x}_{\mathrm{C}}, \mathrm{y}_{\mathrm{C}}$ and the heading angle $\varphi$ showing the deviation of the longitudinal axis of the machine from the coordinate axis Y.

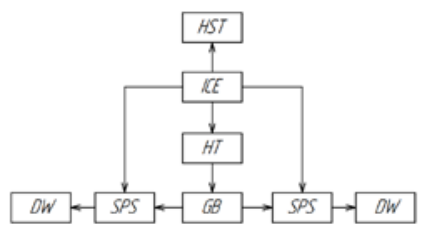

Fig. 1. Kinematic scheme of TV. ICE - internal combustion engine, HST - hydrostatic transmission of the turning mechanism, HT - hydrotransformer, SPS - summing planetary series, GB - gearbox, DW driving wheels
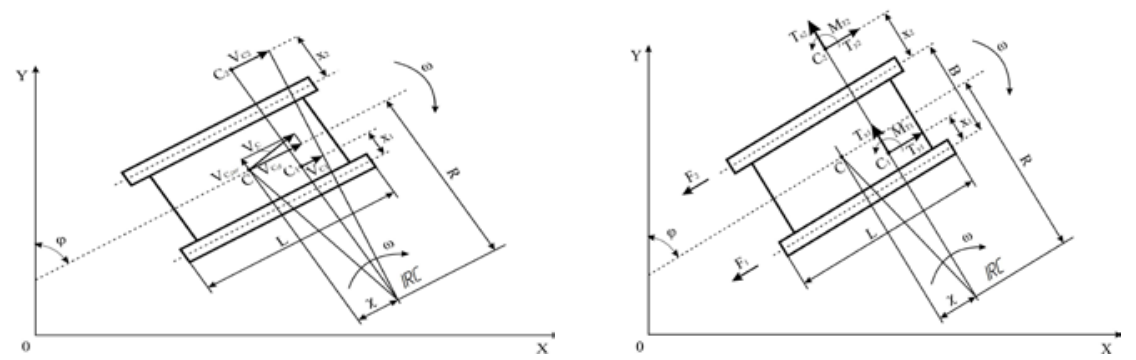

Fig. 2. Calculation scheme: $\mathrm{X}, \mathrm{Y}-$ Cartesian coordinates, $\varphi$ - heading angle, IRC - instantaneous rotation center, $\mathrm{F}_{1}, \mathrm{~F}_{2}$ - resistance forces on the lagging and running board, $\mathrm{V}_{\mathrm{c}}$ - linear velocity of the center of gravity, $\chi$ - longitudinal displacement of the turning pole, $\mathrm{L}$ - longitudinal base of the machine, $\mathrm{B}$ - transverse base of the machine, $\mathrm{x}_{1}$ - transverse displacement of the pole of rotation of the lagging track, $\mathrm{x}_{2}$ - transverse displacement of the turning pole of the running track, $\mathrm{V}_{\mathrm{C} \text { per }}-$ component of $\mathrm{V}_{\mathrm{C}}$, perpendicular to the axis of the machine body, $\mathrm{V}_{\mathrm{C} \text { di }}-$ component of $\mathrm{V}_{\mathrm{C}}$, directed along the axis of the machine body, $\mathrm{T}_{\mathrm{x} 1}, \mathrm{~T}_{\mathrm{y} 1}$ - components of the resulting frictional force of the lagging track to the ground, $\mathrm{T}_{\mathrm{x} 2}, \mathrm{~T}_{\mathrm{y} 2}$ - components of the resulting frictional force of the running track to the ground, $\mathrm{M}_{\mathrm{f} 1}$ , $\mathrm{M}_{\mathrm{f} 2}$ - resulting moments of friction of lagging and running tracks relative to the poles of rotation $\mathrm{C}_{1}$ and $\mathrm{C}_{2}$ correspondently, $\omega=\mathrm{d} \varphi / \mathrm{dt}$ - angular speed of body rotation, $\mathrm{V}_{\mathrm{C} 1}, \mathrm{~V}_{\mathrm{C} 2}$ - the speed of the poles of the rotation of the lagging and running tracks, respectively 
The mathematical model underlying the TV maneuverability studies includes 8 differential equations of the first and second order. Six equations describe the movement of the body of the machine, rotation of the shafts of the engine and the HST motor, the turbine of the hydro transformer (HT). Two equations describe the pressures in the HST pipes [7-9]:

$$
\begin{aligned}
\frac{d^{2} X_{c}}{d T^{2}}= & {\left[\left(P_{2}+P_{1}-P f_{1}-P f_{2}\right) \sin \varphi+R_{\sigma} \cos \varphi\right] \frac{g}{G} } \\
\frac{d^{2} Y_{c}}{d T^{2}}= & {\left[\left(P_{2}+P_{1}-P f_{1}-P f_{2}\right) \cos \varphi-R_{\sigma} \sin \varphi\right] \frac{g}{G} } \\
\frac{d^{2} \varphi}{d T^{2}}= & {\left[\left(P_{2}-P_{1}+P f_{1}-P f_{2}\right) \frac{B}{2}-\operatorname{sign} \frac{d \varphi}{d T} M_{R}\right] \frac{1}{J_{R}} } \\
& \frac{d \omega_{p}}{d T}=\left[M_{E} i_{1}-M_{p}-M_{H P} i_{2}\right] \frac{1}{J_{1}} ; \\
& \frac{d \omega_{w}}{d T}=\left[M_{w}-\frac{\left(P_{2}+P_{1}\right) R_{d w}(\kappa+1)}{i_{g t} i_{g b} \kappa}\right] \frac{1}{J_{2}} ; \\
\frac{d P_{H S T 2}}{d T}= & \left(Q_{m 2}-Q_{p 2}-Q_{k 12}-Q_{k 22}+Q_{k 32}-Q_{k 42}\right) \frac{E}{V_{2}} \\
\frac{d P_{H S T 1}}{d T}= & \left(Q_{p 1}-Q_{m 1}-Q_{k 11}-Q_{k 21}+Q_{k 31}-Q_{k 41}\right) \frac{E}{V_{1}} \\
& {\left[M_{H M}-\frac{\left(P_{2}-P_{1}\right) R_{d w}}{i_{5} \kappa}\right] \frac{1}{J_{3}} ; }
\end{aligned}
$$

\section{Simulation results}

In the process of simulation, the parameters of the tractor's curvilinear motion with a differential rotation mechanism are investigated. The entrance to the turn of the tractor is initiated by a full turn of the helm, for 2 seconds. Since the work of the HST is of particular interest, Fig. $3 \mathrm{a}$ is a graph of the relative (relative to $40 \mathrm{MPa}$ ) pressure in the injection and suction lines. The top graph correspond to the discharge line, lower graph - for the suction line. Since the ground is set to the same, the steady pressures are the same, of the order of 0.6 from $40 \mathrm{MPa}$ to $24 \mathrm{MPa}$. It is important to note that when entering the turn, the pressure exceeds the established pressure by 1.5 times, and this circumstance must be borne in mind, this figure can not be obtained from static calculations, and when testing a real machine, the potential inability to enter the turn can be related with this. 


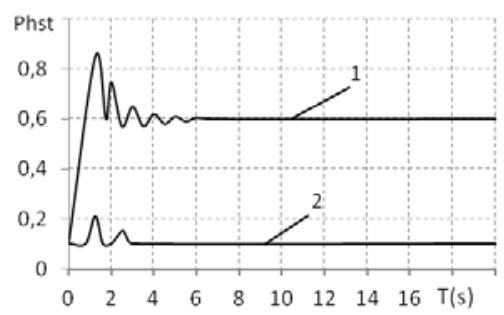

a

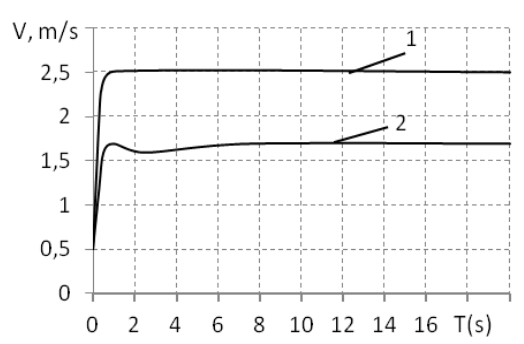

b

Fig. 3. a Relative pressure in the discharge and suction lines; $\mathbf{b}$ The speed of the tractor when working on the ground with the coefficient of resistance $\varphi=0.1$ (1), $\varphi=0.3$ (2)

Further similar motion was investigated, but on a ground with a large resistance (3 times more). Fig. $3 b$ shows the speed of the tractor.

Interesting, but obvious result: the resistance is greater, naturally the HT sags and the speed decreases. Uncertainty in the other: the entrance to the turn is accompanied by a jump in the pressure of the HST, and in the case of less resistance, but greater speed, the jump is much greater, by 15 percent (see Fig. 4a)

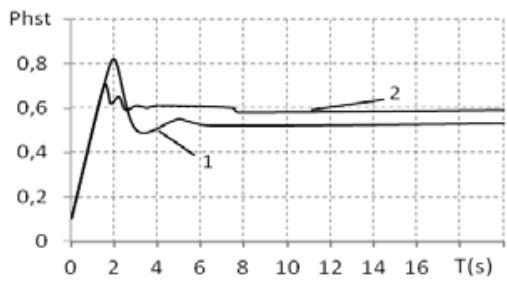

a

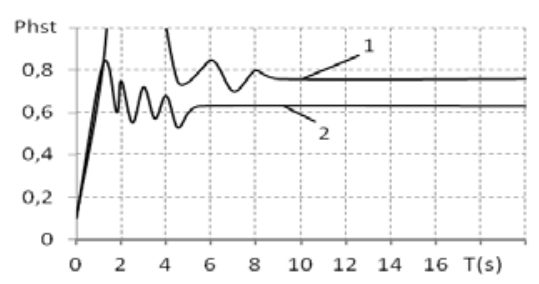

b

Fig. 4. a Pressure in the HST trunks at soil resistance coefficients: $\varphi=0.1$ (1), $\varphi=0.3$ (2); b Pressure in the lines of HST: $1-\mu=0,7, \varphi=0,1 ; 2-\mu=0,7, \varphi=0,3$

When reversing in place, the same picture is the same: for a large resistance, there is a pressure jump (see Fig. 4b).

A lower resistance corresponds to a greater pressure in the transient process, even goes to the valve. The fact is that the thrust force on the running board reaches a limit at $\varphi=0.3$ and does not give the lagging board the necessary negative pull. And this is reflected in the developed angular velocity of the turn at the site, see Fig. 5

Turning on the spot is realized by forces of traction along the sides, or rather the difference between the traction force on the clutch and the resistance of the ground on each side. A lesser resistance leaves a sufficient supply of clutch for the turning moment, loading of the HST and the development of the necessary speed of rotation (turning on the spot). Fig. 5b illustrates the impossibility of achieving a theoretical angular velocity of $0.5 \mathrm{p} / \mathrm{s}$ at a resistance of $\varphi=0.3$

With a resistance of $\varphi=0.1$, a pressure overload (Fig. 4b) occurs due to a sufficient difference in the coefficient of adhesion and resistance, which generates a large angular acceleration of the body of the TV. As a result, the angular velocity of the turn in place achieves the values calculated by the statics $-0.5 \mathrm{p} / \mathrm{s}$. Why not $0.63 \mathrm{p} / \mathrm{s}$ ? Because of skidding of caterpillars about $5 \%$ and losses in HST of about $20 \%$. Both are not taken into account in the statistical calculation. 


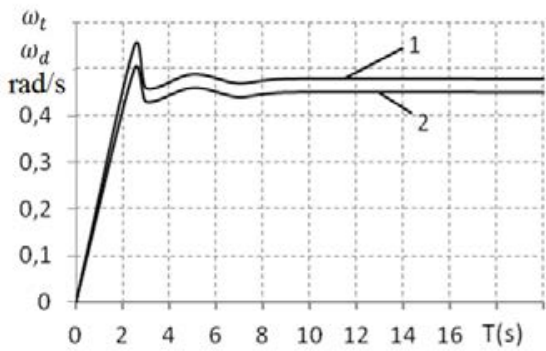

a

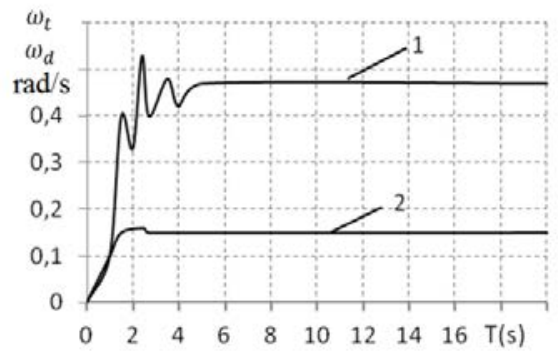

$\mathrm{b}$

Fig. 5. Turning angular velocity in place a) $-\mu=0,7, \varphi=0,1 ; b)-\mu=0,7, \varphi=0,3 ; 1$ - theoretical, according to driving wheels: 2-real, on the ground

\section{Simulation of the tracking system for stabilizing the trajectory of the TV}

There are classic differences in the schemes of the mechanisms for turning American and German tanks and infantry fighting vehicles: in "Abrams" the HST pump is driven from the HT turbine, and in transmission of "Leopard-2" directly from the engine. It is believed that the German scheme has a higher efficiency, since the power expended for the turn does not pass through the HT with its $70 \%$ efficiency. However, there is a significant drawback - with increasing ground resistance, the turbine of HT spontaneously "lands", and the internal combustion engine maintains its rotational speed, which together leads to a reduction in the radius of the trajectory, with the hand wheel being unchanged. In this regard, it is believed that the "Abrams" is managed in the turn better than the "Leopard - 2".

In the scheme under study, the lack of drive of the HST pump from the ICE is evident (see Fig. 1). At time $\mathrm{t}=5 \mathrm{~s}$, the soil resistance coefficient was increased from 0.2 to 0.6 , which led to a spontaneous reduction of the radius from $13.5 \mathrm{~m}$ to $10 \mathrm{~m}$. (Fig 6a). This is due to a decrease in the HT ratio from 0.88 to 0.65 .

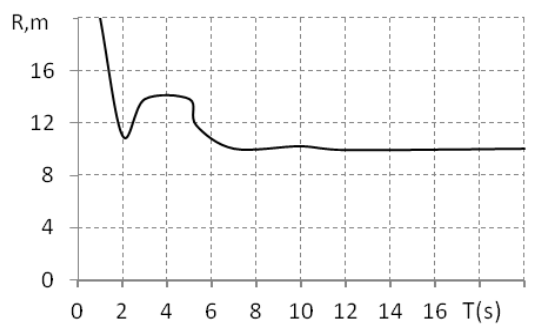

a

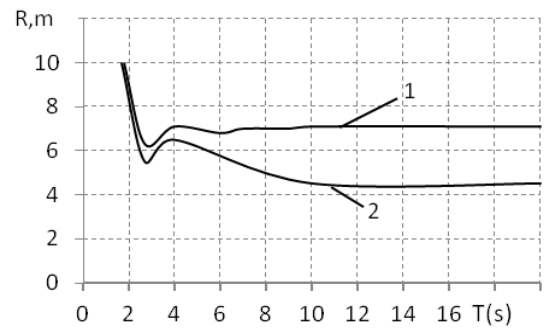

b

Fig. 6. a Radius of the trajectory of the motion of the TV with increasing resistance from $\varphi=0.1$ to $\varphi$ $=0.3$ at a time of $5 \mathrm{sec}$.; $\mathbf{b}$ Radius of the trajectory of the tractor with gradual soil set: 1-equipped with a tracking system; 2 - without tracking system

The authors proposed to add additional information (on the speed of rotation of the turbine of HT) to the controller that controls the supply of the HST pump and to correct the feed of the HST pump regardless of the position of the hand wheel. 
When bulldozing a single jump in the disturbance is possible when the obstacle is hit in the form of a stone in the ground. More often, resistance increases gradually as the soil is collected in the prism. The influence of the ground set process on the tractor, not equipped with a tracking system and equipped with it, is estimated. Comparative results are shown in Fig. 6b. The graphs in Fig. 6b show that when bulldozing in a turn, the trajectory radius of a tractor, not equipped with a tracking system, spontaneously decreases $6 \mathrm{~m}$ to $4.5 \mathrm{~m}$, that is, by $25 \%$. Equipping the tractor with a tracking system will stabilize the curved path, make it independent of the changing external load.

\section{Conclusions}

1. The mathematical model of the movement of a tracking vehicle is developed by introducing a new circuit for connecting power currents in the transmission and rotation mechanism of a caterpillar tractor and a model of a tracking system for maintaining a stable radius with the steer.

2. It is established that when entering the turn at the highest gear, the pressure in the HST of the turning mechanism is half the time that the steady-state value.

3 . When entering the turn on heavy ground, there is not enough grip on the ground for maneuvering.

4. At the beginning of the turn on the ground on the ground with a coefficient of resistance to the turn of more than 0.7 , the pressure in the HST line reaches $40 \mathrm{MPa}$.

5. An algorithm for additional control of the supply of a HST pump is proposed, which makes it possible to preserve the curvature of the trajectory with increasing resistance.

The work has been performed with the financial support of the Ministry of Science and Higher Education of the Russian Federation as a part of complex project to create a high-tech production "Development of a stepless differential steering mechanism with tracking control system for new generation offroad and road-building machines" under the agreement No. 074-11-2018-006 d.d. May, 31, 2018 between the Ministry of Education and Science of the Russian Federation and Manufacturing company "Khodovye systemy" in cooperation with the head executor "NIOKTR" - Federal State Autonomous Educational Institution of Higher Education "South Ural State University (National Research University)".

\section{References}

1. Ed. by E.S. Kistochkin, Volumetric hydromechanical transmissions (Leningrad: Mechanical Engineering, 1987)

2. V.A. Petrov Hydraulic transmission of transport vehicles (Moscow Mechanical Engineering, 1988)

3. N.A. Zabavnikov Fundamentals of the theory of transport caterpillar vehicles (Moscow: Mashinostroenie, 1968)

4. P.P. Isakov, Theory and construction. - V. 5. Transmission ( Leningrad: Mechanical Engineering, 1985)

5. Yu.V. Ginzburg, A.I. Shved, A.P. Parfenov, Industrial tractors (Moscow: Mechanical Engineering, 1986)

6. M.I. Zlotnik, I.S. Kaviarov, Transmissions of modern industrial tractors (Moscow: Mechanical Engineering, 1971) 
7. S.V. Kondakov, D.V. Kharlapanov, E.I. Vansovich, Models of the Turn Resistance for High-Speed Caterpillar Vehicles, Russian Engineering Research, v. 36(1), pp. 1-5 (2016)

8. S.V. Kondakov, O.O. Pavlovskaya, Automated control of movement of a high-speed tracked vehicle: monograph (Saarbrucken: LAP LAMBERT Academic Publishing, 2013).

9. S.V. Kondakov, E.I. Vansovich, Increase of controllability of the high-speed caterpillar by creating a double-flow hydrostatic-mechanical differential turning mechanism, Vestnik SUSU. Series "Mechanical Engineering" v. 19 (12 (271)), pp. 117-123 (2012) 